\title{
Prevalence of Vitamin B12 Deficiency In Patients With Type II Diabetes Mellitus On Metformin
}

\author{
Malla D, Bajracharya MR, Karki BB, Rajouria AD, Shrestha PS \\ National Academy of Medical Sciences, Bir Hospital, Kathmandu, Nepal
}

\begin{abstract}
Background: Diabetes Mellitus is one of the most encountered disease in our out patient department and metformin is the first drug of choice to treat Diabetes mellitus. As metformin is one of the cheapest drug many patients use these drug for long period of time with consultation and without consultations with doctors. Patients under long term metformin use are not aware of Vitamin B12 deficiency and its associated signs and symptoms. In Nepal due to poverty, lack of education and awareness on diabetes mellitus we doctors find much difficult to explain patients on the consequences of diseases. So I decided to do this study which could be much easier to explain patients on effect of metformin of vitamin B12 levels and the consequences life style modifications and supplement of Vitamin B12 to the patients. Methods: This is a Cross-Sectional Study done in the patients with Type 2 diabetes were selected based on inclusion and exclusion criteria. Basic biochemical investigation were send the lab of the National academy of medical science. Serum B12 assay were done. Vitamin B12 deficiency is defined as values $<150 \mathrm{pg} / \mathrm{ml}$. Association between vitamin B12 deficiency with duration of metformin therapy, duration of diabetes, with age, sex were done. Results: The mean vitamin B12 level is low as the duration of metformin treatment increases. The sex, age relation with development of vitamin B12 deficiency was not significant. In my study out of 210 patients 107 patients were having severe vitamin B12 deficiency level and 63 patients had a borderline Vitamin B12 deficiency level which shows that the deficiency increases as per longer use of metformin, which shows prevalence of 50.95\%. Conclusions: Vitamin B12 deficiency occurs in type 2 diabetes mellitus patients treated with long term metformin. The duration of metformin therapy significantly affects the development of vitamin B12 deficiency. As a treating physician we always need to explain our patients about the side effect of metformin and regular follow up and investigations must be done to early diagnosis of vitamin B12 deficiency to improve the quality of life.
\end{abstract}

Keywords: B12 deficiency, Diabetes Mellitus, Metformin.

\section{Introduction}

Metformin has long been considered the initial drug therapy choice in the treatment of type 2 diabetes mellitus (T2DM). When monotherapy is initially preferred to treat hyperglycemia, Metformin is the widely recommended and violet et al in 2012 mentioned that it is prescribed to 120 million patients with diabetes around the world ${ }^{1-5}$.

Correspondence Author

Dr. Dipak Malla, Assistant Professor

Department of Internal medicine, National Academy of

Medical Sciences
It was in 1971, Tomkin et al first wrote an article describing Metformin associated vitamin B12 malabsorbtion. ${ }^{6}$ Metformin lowers Vitamin B12 level and is associated with Vitamin B12 deficiency has been document in various studies in the past. ${ }^{7-}$ ${ }^{14}$.How Metformin causes Vitamin B12 deficiency is not clear but it is said that it stimulate the small bowel bacterial overgrowth and by directly decreasing vitamin B12 absorption. Lui et all in 2006 and Toh et all in 2009 also stated same with one of the documented side effects of metformin is vitamin B12 deficiency but it is almost always overlooked and seldom investigated ${ }^{15-16}$. Metformin 
also alters the intracellular handling of calcium on terminal ileum which decreases the absorption of the vitamin B12 intrinsic factor complex, which is later supported by a study done by Bauman et al 2000 that administration of the calcium reverses metformin induced vitamin B12 deficiency. ${ }^{17}$

Most of the time vitamin B 12 deficiency was not thought in such clinical situation. There is no universal recommendation to supplement vitamin B12, especially in high risk populations. Studying the biochemical profile to detect vitamin B12 deficiency in these populations will provide useful data to support the need for supplementation. The Objectives was to assess the Vitamin B12 level in chronic metformin treated type 2 diabetic patients and to assess the relation between metformin therapy duration \& development of vitamin B12 deficiency.

\section{Materials and Methods}

The patients included in this study were from the National academy of medical sciences from September 2018 to Feb 2019. This was a Cross sectional study done in Diabetic outpatient department, National Academy of Medical Sciences, Bir Hospital. The inclusion criteria was Type 2 Diabetic patients, who were on Metformin therapy for more than 18 months. The present study will be conducted at National academy of medical sciences, Bir Hospital, kathmandu, which is a tertiary care center. This study will screen around 263 patients both in-patients and out patients with T2DM on metformin therapy for Vitamin B12 deficiency. This study was done to examine the prevalence of vitamin B12 deficiency in patients with T2DM on metformin attending National academy of Medical sciences,Bir Hospital.

The Exclusion Criteria was the Patient diagnosed with Pernicious anaemia, Malabsorbtion (coelic disease, Inflammatory disease, gastrointestinal surgery), Alcoholism, Malnutrition, Iron deficiency anaemia, history of thyroid disease and on thyroxine treatment and or a history of other organ specific autoimmune conditions(Vitiligo, addisons disease,
Hypoparathyroidism), Surgery involving small intestine or HIV infection. Intake of vitamin b12 or any multivitamin preparation during past 6 months

\section{Results}

Our study comprised of 263 type 2 diabetic population. Out of which 153 are males \& 111 are females. The number of years patients were on metformin use is shown in Fig 1.

Fig 1: shows total number of years the patients

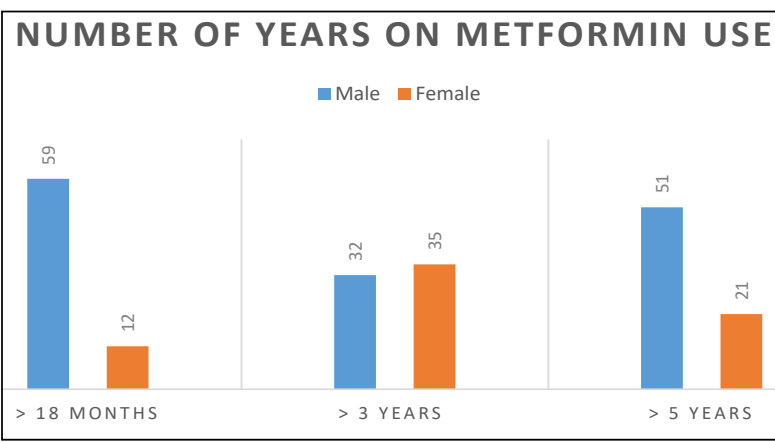

were on metformin use, which shows that as the number of year increases patients are using more Metformin.

Among the study groups, the level of vitamin B12 among the male and females are shown in Fig 2. The relation between vitamin b12 deficiency and number of years on metformin use are shown in fig 3 . Fig 2: Chart shows the level of Vitamin B12 levels

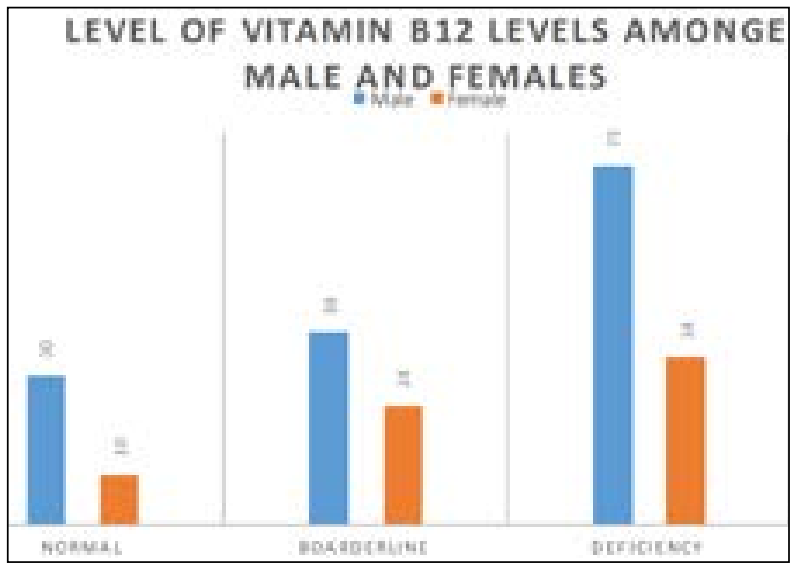

among male and female patients

Fig 3: Chart shows relation between Vitamin $B 12$ 


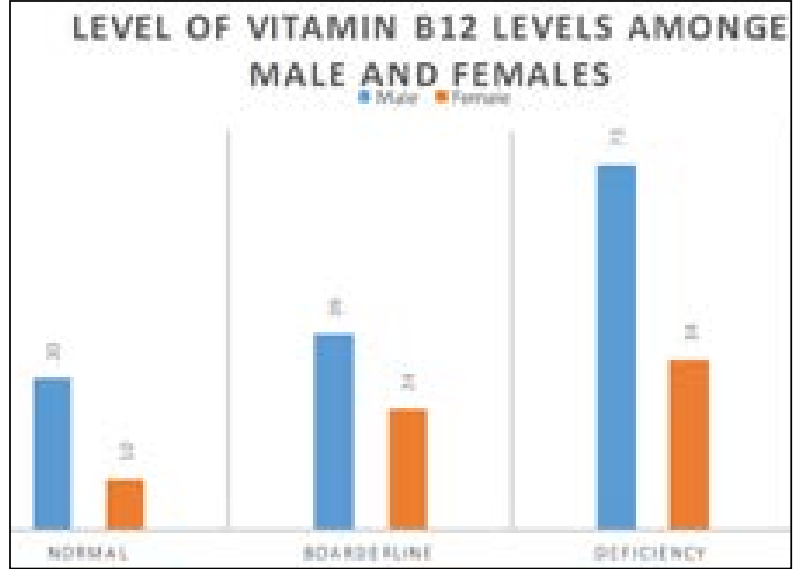

deficiency and number of years the patients using Metformin

Fig 4: Chart shows relation between dose of

\section{RELATION BETWEEN DOSE OF METFORMIN} AND VITAMIN B12 DEFICIENCY

$\because 500 \mathrm{mg} B \quad \square 1 \mathrm{gm} \mathrm{BD}$

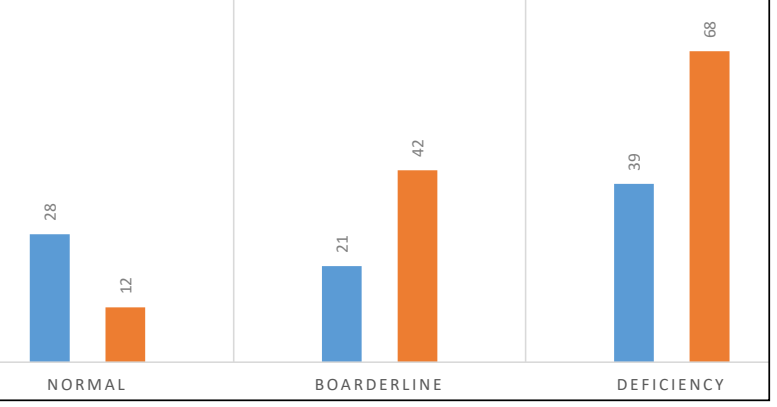

Metformin and Vitamin B12 levels

\section{Discussion}

Metformin being the most commonly prescribed drug for the treatment of diabetes mellitus and there are proposed mechanism through which metformin interacts with vitamin B12 absorption, it causes deficiency of vitamin B12. It is a cross sectional study where the serum B12 level of type 2 Diabetic patients who are on metformin therapy (18 months) was measured and correlated with duration of metformin therapy, duration of diabetes mellitus.

After exclusion criteria as per use of Vitamin
B12, Patients with CKD,Iron deficiency Anemia, long use of Alcohol use, past and recent history of Thyroid illness and past Gastrointestinal surgery 54 patients were excluded from the study. So 210 patients as 142 male and 68 females were included in the study.

71 patients were using metformin for more than 18 months, 67 for more than 3 years and 72 for more than 5 years, shows that metformin use also increases according to the increase in number of years of Diabetes.

In my study 107 patients were having severe vitamin B12 deficiency level and 63 patients had borderline Vitamin B12 deficiency levels which shows that the deficiency increases as per longer use of metformin. This value shows the prevalence of $50.95 \%$ with vitamin B12 deficiency amonge patients on long term metformin use. Our study is also being supported by ting et $\mathrm{al}^{24}$ and pierce et $\mathrm{al}^{25}$. with this results of my study I am much aware that care full followup of the patients with metformin on both high dose and low dose in very important and I must advice my patients for annually check up vitamin B 12 level,that I was not aware or is being neglected before due to poverty and irregular follow up of patients.

This study also shows that the dose of metformin also effects the level of Vitamin B12.As seen in our results 68 patients with severe Vitamin B12 were on Metformin 1gm on twice daily basis. Our study is also being supported by ting et al. ${ }^{24}$

As we see increased prevalence of vitamin B12 deficiency,we can also recommend annual screening for vitamin B12 and supplementation should be adopted among diabetic patients with specific risk factors of vitamin B12 deficiency which is also supported by kribirige $\mathrm{D}$ and Mwebeze $\mathrm{R}$ in $2013 .^{26}$

The prevalence of Vitamin B12 defiency in Patients with T2DM on metformin therapy in Nepal is unknown and the measurement of serum Vitamin $\mathrm{B} 12$ in T2DM is not a part of the standard annual 
review examination. Many studies were done in the past and showed that prevalence of Vitamin B12 deficiency varied greatly and ranged between $5.8 \%$ and $52 \% .^{27-35}$

\section{Conclusion}

In a country like Nepal where most of the patients coming to our out patient department are from remote areas of Nepal, we as a treating physician must explain our patients about the possible side effects of Metformin, which is one of the mostly used drug and patients use metformin for long period of time without consulting the doctors. Chronic use of metformin causes symptomatic and asymptomatic vitamin b12 deficiency.

Vitamin B12 assay in the patients who were on chronic metformin therapy helps in assessing Vitamin B12 deficiency.

The duration of metformin therapy has affected the vitaminB12 status significantly.

\section{References:}

1. Viollet B, Guigas B, Sanz Garcia N, Leclerc J, Foretz M, Andreelli F. Cellular and molecular mechanisms of metformin: an overview. Clin Sci (Lond). 2012;122(6):253-70.

2. International Diabetes Federation. Global guideline for type 2 diabetes. Available from: http://www.idf.org/global-guideline-type-2diabetes2012. Accessed September 5, 2013.

3. National Institute for Health and Clinical Excellence. Type 2 diabetes: The management of type 2 diabetes (NICE clinical guideline 87). Available from: http://www.nice.org.uk/ guidance/CG87. Accessed August 15, 2013.

4. Inzucchi SE, Bergenstal RM, Buse JB, et al. Management of hyperglycemia in type 2 diabetes: a patient-centered approach. Diabetes Care. 2012;35:1364-1379.

5. Garber AJ, Abrahamson MJ, Barzilay JI, et al. American Association of Clinical Endocrinologists' comprehensive diabetes management algorithm 2013 consensus statement. Endocr Pract. 2013;19 Suppl 2.
6. Filioussi K, Bonovas S, Katsaros T. Should we screen diabetic patients using biguanides for megaloblastic anaemia? Aust Fam Physician 2003;32: 383- 4 .

7. Pongchaidecha M, Srikusalanukul V, Chattananon A, Tanjariyaporn S. Effect of metformin on plasma homocysteine, vitamin B12 and folic acid: a crosssectional study in patients with type 2 diabetes mellitus. J Med Assoc Thai 2004;87:780 -7.

8. DeFronzo RA, Goodman AM. Efficacy of metformin in patients with non-insulindependent diabetes mellitus. N Engl J Med 1995;333:541-9.

9. Hermann LS, Nilsson B, Wettre S. Vitamin B12 status of patients treated with metformin: a crosssectional cohort study. Br J Diabetes Vasc Dis 2004; 4:401-6.

10. Andre's E, Noel E, Goichot B. Metforminassociated vitamin B12 deficiency Arch Intern Med 2002;162: 2251-2.

11. Wulffele' MG, Kooy A, Lehert P, et al. Effects of short-term treatment with metformin on serum concentrations of homocysteine, folate and vitamin B12 in type 2 diabetes mellitus: a randomized, placebocontrolled trial. J Intern Med 2003;254:455- 63.

12. Tomkin GH, Hadden DR, Weaver JA, Montgomery DA. Vitamin- B12 status of patients on long-term metformin therapy. $\mathrm{Br}$ Med J 1971;2:685-7.

13. Stowers JM, Smith OA. Vitamin B12 and metformin. Br Med J 1971;3:246 -7.

14. Liu KW, Dai LK, Jean W. Metformin related vitamin B12 deficiency. Age and Ageing. 2006; 35(2):200-1.

15. Toh SY, Zarshenas N, Jorgensen J. Prevalence of nutrient deficiencies in bariatric patients. Nutrition. 2009; 25:1150.

16. Bauman WA, Shaw S, Jayatilleke E, Spungen AM, Herbert V. Increased intake of calcium reverses vitamin B12 malabsorption induced by metformin. Diabetes Care. 2000 Sep;23(9):1227-31. 
17. Ting RZ, Szeto CC, Chan MH, Ma KK, Chow KM. Risk factors of vitamin B12 deficiency in patients receiving metformin. Arch Internal Med. 2006;166:1975-9.

18. Pierce S, Chung A, Black K (2012) Evaluation of Vitamin B12 Monitoring in a Veteran Population on Long-Term, High-Dose Metformin Therapy. Ann Pharmacother 46:1470-1476.

19. Kibirig Mwebaze R. Vitamin B12 deficiency among patients with diabetes mellitus: is routine screening, and supplementation justified? J Diabetes Metab Disord. 2013 May 7;12(1):17. doi: 10.1186/2251-6581-12-17.

20. de Jager J, Kooy A, Lehert P, Wulffele MG, van der Kolk J, Bets D, et al. Long term treatment with metformin in patients with type 2 diabetes and risk of vitamin B12 deficiency: randomised placebo controlled trial. BMJ. 2010;340:c2181.

21. Reinstatler L, Qi YP, Williamson RS, Garn JV, Oakley Jr GP. Association of biochemical B12 deficiency with metformin therapy and vitamin B12 supplements: the National Health and Nutrition Examination Survey, 1999-2006. Diabetes Care. 2012;35(2):327-33.

22. Hermann LS, Nilsson B, Wettre S. Vitamin B12 status of patients treated with metformin: a cross-sectional cohort study. Br J Diabetes Vasc Dis. 2004;4:401-4.
23. Liu KW, Dai DL, Ho W, Lau E, Woo J. Metformin-associated vitamin B12 deficiency in the elderly. Asian $\mathrm{J}$ Gerontol Geriatr. 2011;6:82-7.

24. Singh AK, Kumar A, Karmakar D, Jha RK. Association of B12 deficiency and clinical neuropathy with metformin use in type 2 diabetes patients. J Postgrad Med. 2013;59(4):253-7.

25. de Groot-Kamphuis DM, van Dijk PR, Groenier KH, Houweling ST, Bilo HJ, Kleefstra N. Vitamin B12 deficiency and the lack of its consequences in type 2 diabetes patients using metformin. Neth J Med. 2013;71(7):386-90.

26. Calvo Romero JM, Ramiro Lozano JM. Vitamin $\mathrm{B}(12)$ in type 2 diabetic patients treated with metformin. Endocrinol Nutr. 2012;59(8):48790.

27. Kang D, Yun JS, Ko SH, Lim TS, Ahn YB, Park YM, et al. Higher prevalence of metformin-induced vitamin B12 deficiency in sulfonylurea combination compared with insulin combination in patients with type 2 diabetes: a cross-sectional study. PLoS One. 2014;9(10):e109878.

28. Beulens JW, Hart HE, Kuijs R, KooijmanBuiting AM, Rutten GE. Influence of duration and dose of metformin on cobalamin deficiency in type 2 diabetes patients using metformin. Acta Diabetol. 2014;52(1):47-53. doi: 10.1007/s00592-014-0597-8. 